\title{
A New Framework for the Evaluation of QoS in Cloud Federation
}

\author{
Ali Ghazi \\ Department of Computer \\ Engineering, Mahallat Branch, \\ Islamic Azad University, \\ Mahallat, Iran
}

\author{
Hamideh Babaei \\ Department of Computer \\ Engineering, Naragh Branch, \\ Islamic Azad University, \\ Naragh, Iran
}

\author{
Mostafa Ghobaei Arani \\ Department of Computer \\ Engineering, Parand Branch, \\ Islamic Azad University, \\ Tehran, Iran
}

\begin{abstract}
In cloud computing it is assumed that a single cloud with increased service demands because of the limitations of the hardware and software resources, can encounter overload. Cloud Federation is an ideal solution to overcome these problems. There are different types of single and hybrid clouds in Cloud Federation. Also, one of the aspects of union activity is based on borrowing hardware and software resources and other cloud computing to reduce and stop the over load of host cloud. Therefore, measuring the quality of a single cloud service and hybrid cloud service in cloud federation carries much importance. To this end, the models based on the initial model were used to measure the Quality of Service .The present paper by providing an appropriate framework through a dynamic measuring mechanism calculates service quality parameters like average failure rate and reliability for each cloud service and then for root hybrid cloud service. The simulation results of this measurement mechanism compared to the type of cloud service connectivity in hybrid cloud service shows improvement in the parameters of the quality of hybrid cloud service.
\end{abstract}

\section{Keywords}

Cloud computing, cloud federation, Quality of Service, hybrid cloud service, continuous measurement.

\section{INTRODUCTION}

Today, cloud computing is one of the most challenging research topics in IT and the use of this technology is growing day by day. Cloud computing means developing and implementing computer technology based on the Internet. A variety of providers for providing service to users due to various reasons such as cost and performance are connected to each other. Thus hybrid services are in contact, interact and sometimes in contrast with other services and encounter with numerous challenges. In order to solve security challenges, control intellectual property, standardize service interfaces, define protocols and homogenize data formats as well as provide various types of hybrid services by various providers the necessity of using cloud computing concept is very important in the development of cloud federation. To provide good quality service for users and efficient use of resources and IaaS, it is necessary to examine the various aspects of Cloud federation. The concept of cloud federation due to partial outsourcing and sharing parts of resources which further reduce costs is much more affordable. Thus, cloud federation provides new vision of the legal aspects and also security and quality requirements. IaaS, PaaS and SaaS are different levels of service providing in cloud computing thus in the architecture of cloud federation, new services are modeled vertically and horizontally. In the horizontal model service is provided on one level while vertical service is multilevel [1].

Thus, given the diversity of clouds, plurality of providers and the importance of observing the obligations in service level agreement (SLA), one of the challenges posed by cloud computing is to evaluate the Quality of Service (QoS) for commercial applications in such systems. Evaluating Quality of Service parameters and measuring service quality levels and identify threshold levels, can provide a SLA management processes .Availability, processing time, the way of distribution of task on different clouds, accuracy of results, safety and cost are among effective parameters in measuring and assessing the quality of services [2]. Different models have been studied for evaluating Quality of Service in the clouds and cloud federation. Due to the extensive variety of services, clouds and IaaS of each strategy have a special range of assessment and do not fully cover all the effective components in the Quality of Service [3, 4]. Some of the patterns are evaluated based on tree structure that hybrid service tree is achieved after the generation of sub-trees, doing related processes and compounding them [5]. Also another research studied five strategies of computational redundancy and their effect on availability, time processing, task distribution among different clouds, accuracy of results, safety and cost. Then the quality level of cloud was measured and after that the quality level in Cloud federation has been analyzed according to each strategy in cloud federation. Also they have been analyzed and explained the different trade-offs and effective parameters on the Quality of Service among these strategies in [6]. Another investigative approach by providing System of Systems (SoS) approach to evaluate the Quality of Service has studied the evaluation in cloud environments. SoS has a hierarchical structure in which oversight and accountability are at higher levels and smaller components of the system such as bit are at lower [7]. To assess the efficiency of services in cloud computing systems, various models based on queuing theory has been studied [8]. Also, some studies have proposed a semantic -based model that has a broker [9]. Also, some of them have used a fuzzy logic approach to secure QoS and guarantee SLA level of services from the change in size and scale to the demand in the cloud IaaS layer [10].

\section{CLOUD FEDERATION}

Cloud computing and the basic definitions of these categories are described in detail in many books $[11,12,13]$. Here only a part of it is mentioned. 


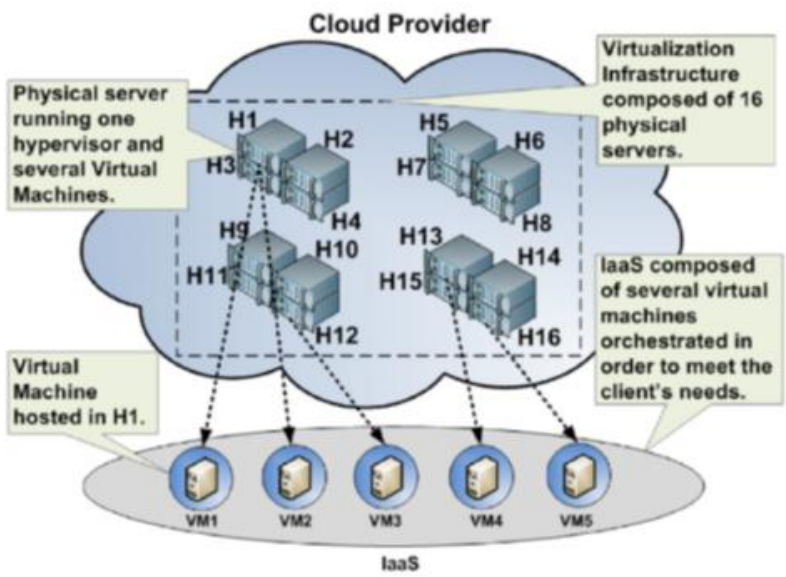

Figure 1: provided resources by IaaS based on user needs[9]

Cloud computing has a concentrated control on the resources that are connected to each other in data centers and the service is provided under the management of a provider. Therefore, cloud computing has some economic benefits, increased productivity of resources as well as reduce costs of IT management.

In cloud computing service models can [2] can be distinguished from each other in the form of IaaS as a service, Platform as a Service and Software as a service. IaaS is based on users' needs and in PaaS 15 complementary platforms related to services and execution environment are provided while SaaS is a model of software providing. Cloud federation include services of several different provider that are accumulated in a tank to support three basic characteristics of interoperability, including resource migration, resource redundancy and the combination of complementary resources and services. Migration is the mobility of resources such as virtual machines, data items, source code, etc. from more than one service domain to another domain, while the redundancy of resources makes possible the simultaneous use of the same service characteristics in different domains and the combination of resources and complementary services makes possible the combination of a variety of services with together. Cloud federation is useful for providers as well as customers. Consumers benefit from higher efficiency and lower costs, while providers offer more sophisticated services. Anyway, from now on we'll focus on the customer perspective. Cloud federation can be distinguished from several different aspects:

A) Federation occurs within a technical layer of the cloud stack (i.e. uniting two services at the IaaS level) or between layers (for example the combination of a platform as a (PaaS) service and one infrastructure as a service (IaaS).

B) Cloud federation is presented in two dimensions: horizontal and vertical. Horizontal federations occur on a surface of the cloud stack. For example, the integration of services at the application level or the integration at the level of infrastructure, as figure (2) all infrastructure service vendors and providers can interact and communicate in IaaS layer. So based on laws and agreements through sharing of storage resources or excess available computing and processing resources can provide the needed resources of other suppliers to enable them to provide the desired services to their users and patrons. Vertical federations have several levels and can occur between layers. Following some features of horizontal federations are considered.

C) To establish federation within the layers of infrastructure as a (IaaS) provider service, there are two main types of services: computing service and storage service.

D) In cloud federation making relations and communications can occur for an indefinite period (permanent) or for a limited period of time (temporary).

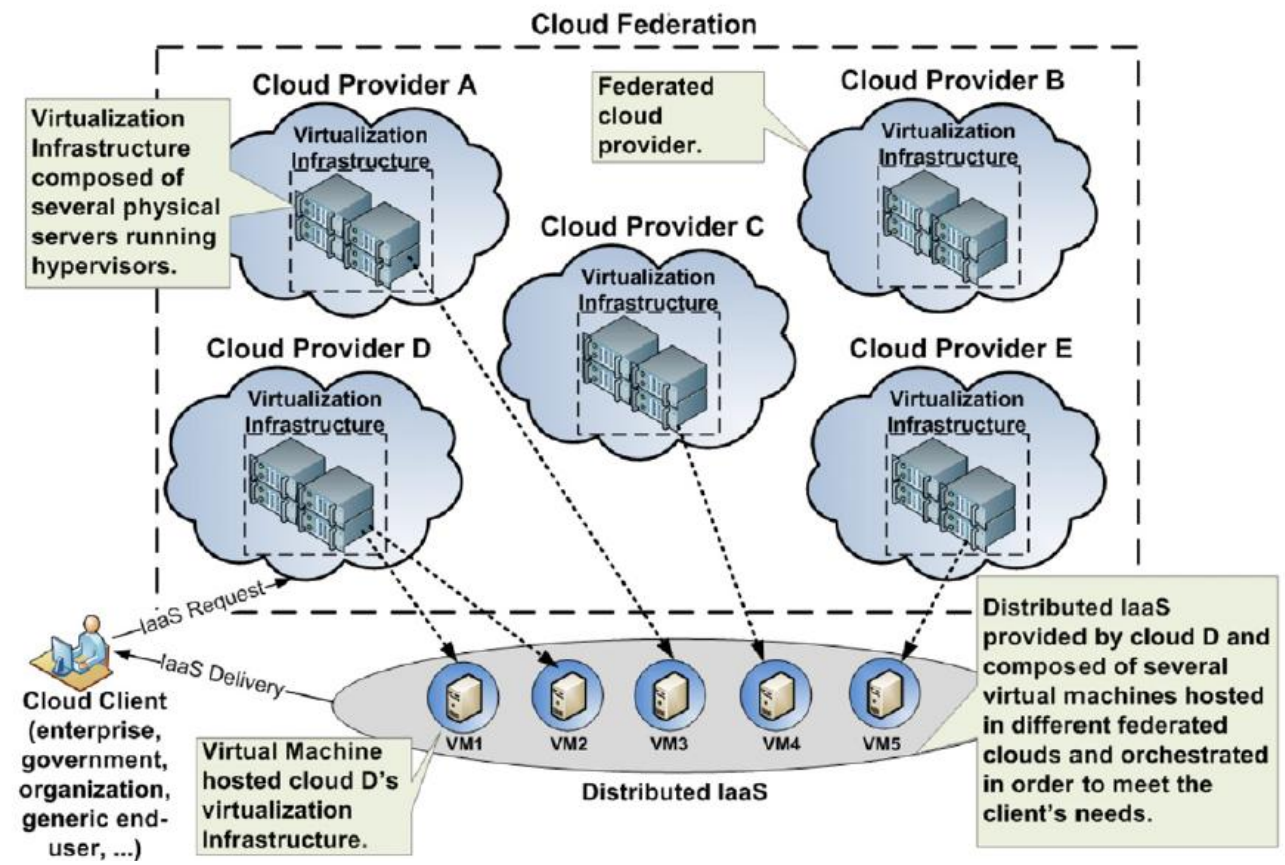

Figure2: Horizontal federation in infrastructure layer 
Horizontal federation can be useful and distinct from several aspects, for example, it may reduce the dependency of provider to domain and other geographical domain and but can activate through the use of other providers across the domains. Thus, through horizontal federation the risk for provider reduces while the increased availability may be obtained throughout several zones. In a continuous cloudy environment or cloud federation, service may be formed by other services which belong to different clouds with different cloud providers. So based on the mutual contracts and agreements, providers should respect the minimum or greater than the service quality level of their consumers. A key challenge for service providers will be to demonstrate QoS with the required level of agreement. A major challenge in cloud computing is unpredictable performance, because providers are unable to anticipate transient changes in demand for services and changes in the geographical distribution of consumers. Furthermore, no single cloud provider is able to create a large enough organizational IaaS that include the concept of unlimited computing resources of cloud computing examples. Cloud federation facilitates the dynamic development and integration of software services among several clouds to achieve the QoS aims with changing workload and computing resources. Due to the dynamic nature of cloud federation, continues monitoring of QoS features are necessary to implement service level agreements. Several research activities have been conducted on the quality of services in cloud federation and researchers have reviewed and provided different approaches to calculate the effective parameters in the quality of service.

\section{NEW FRAMEWORK FOR EVALUATING OF QOS IN CLOUD FEDERATION}

There is a mechanism for continuous measurement of QoS in a cloud federation in which sources are reviewed based on compliance or non-compliance with conditions and rules in service level agreement (SLA). In QoS measurements, there are single cloud services for each one day object service that are related to the arrangement of 1440 minutes that each section shows one minute of the day. By default, every minute is specified with the "UP" or "1". When during a particular one minute a service disruption occurs this minute is specified as "DWN" or "0". Furthermore, as a sign to show the minutes that the user is not concerned with service "Up" or "Down", $\times$ is used. Until the estimated QoS is greater than or equal to target QoS and up to that minute service reaches its goal. In the service tree, when a child node has a bottleneck, motherservice for each "down" minute of this bottleneck suffers an impairment, however, if some redundents are made absolutely one or two of the servers will not affect the service.

In order to have the availability of hybrid services, all main services should be available simultaneously. This mechanism uses intersection to identify the minutes the hybrid service is available.

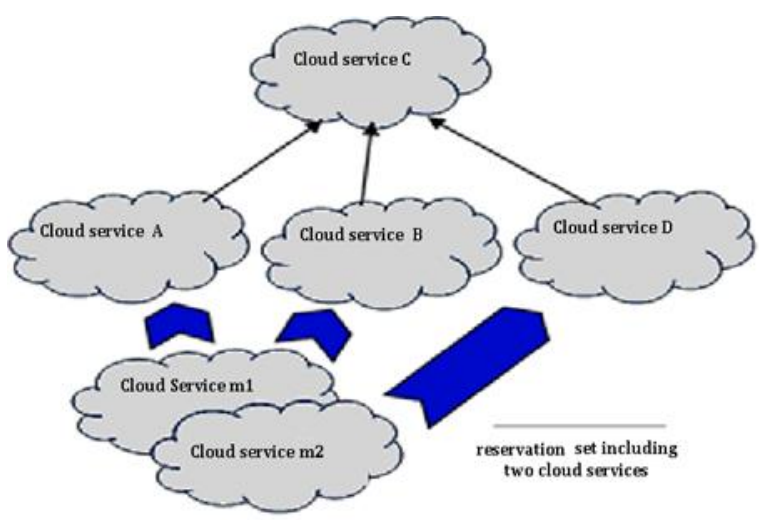

Figure 3: Hybrid cloud service $\mathbf{C}$ with three sub-clouds A, $B$ and $D$ and two cold cloud service reservations

In cloud federation some single or even hybrid cloud services due to having free resources can make their resource available to other cloud services. Primarily, in cloud federation for each hybrid cloud service according to the type of service and its sub- clouds there is a set of free resources of cloud services that are considered as cloud service reservation. The type of cloud service reservation can be hot, warm or cold. In hot reservation, the cloud service reservation activates along with the corresponding sub- cloud service and synchronization data is transmitted between them, this is very similar to the redundant sub- clouds. In warm reservation only sometimes synchronization data is transmitted and is ready to activate for the root cloud service. In cold reservation of cloud service no cloud is active and no synchronization data is transferred between them. Only in the case of failure in synchronization data and information resources, a normal active sub-service is transferred to the cloud service reservation. Then cloud service reservation connects to the root service cloud to transfer the results to the root and therefore activates cloud service reservation. As shown in Figure (3) hybrid cloud service $\mathrm{C}$ has three sub-clouds i.e. A, B and D that two cold cloud service reservations support them.

Hybrid cloud service C in Figure (3) with any failure, subservice uses a sub-cloud service reservation and finally with the occurrence of 2 failures both of the two cloud service reservations are replaced and with occurrence of any failure each sub-cloud service reservation like pervious sub-cloud services is considered as a redundant. In this connectivity model in order to theoretically calculate the reliability and other failure parameters of root cloud service, Markov chain was used which is going to be described in following paragraphs. To evaluate root hybrid cloud service, here 2 redundant active sub-clouds and 1 cold reservation sub- cloud is used. According to Figure (4), to theoretically calculate the reliability and other failure parameters of hybrid cloud service with 2 redundant and one cold cloud reservation Markov chain is used. 


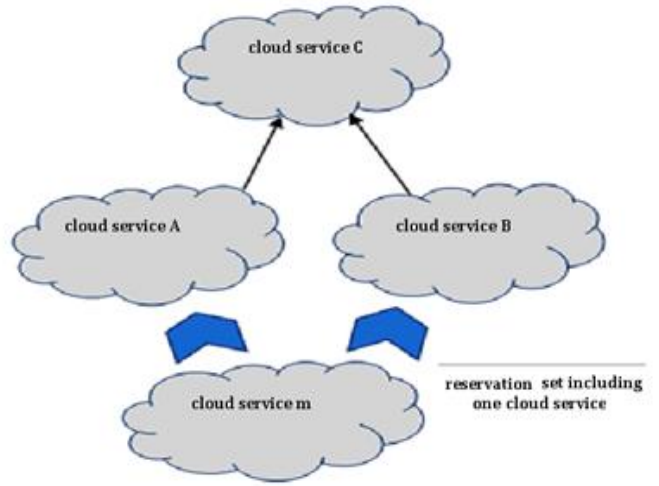

Figure 4: Hybrid cloud service $C$ with redundant subclouds A, B and one cold cloud service reservation

If the failure rate is equal to $\lambda$ and same for all clouds then the graph of Markov chain of hybrid cloud service $\mathrm{C}$ with redundant sub-clouds A, B and cold cloud service reservation $\mathrm{m}$ is as figure (5).This Markov chain has 5 modes that each has two numbers is the number of the left side shows normal and active cloud services and the right number shows the number of cloud service reservation. Hybrid cloud service starts its activity from 2,1 mode therefore in this case both of the sub- cloud services A and B are active and sound and there is also a sound cloud service reservation. The failure rate in both sub- cloud services of A and B is equal to $\lambda$ but in the case of the failure, one of the sub- cloudy services with the probability of $2 \lambda \Delta \mathrm{t}$ transits in to the 1,1 mode which in this case, one cloud service is active and sound and there is one cloud service reservation that is still active and has not been replaced by the defected cloud service. As is clear from Figure (5) there is two modes of 2,0 and F of 1,1 mode. If, only the active sub-cloud service defects with the probability of $\lambda \Delta t$, hybrid cloud service defects that is the F mode, and if the cloud service reservation activates with the possibility of $\delta \Delta \mathrm{t}$ then 2.0 occurs. Based on the figure, the model has two levels and 1,1 step is the first level (from the top) that its failure sub-cloud service and out let is not detected yet. In second level cloud service was detected defective and with the possibility of $\delta \Delta \mathrm{t}$ went out of service and was replaced with sub-cloud service reservation. Modes 1,0 and $F$ are the repetition of first level modes.

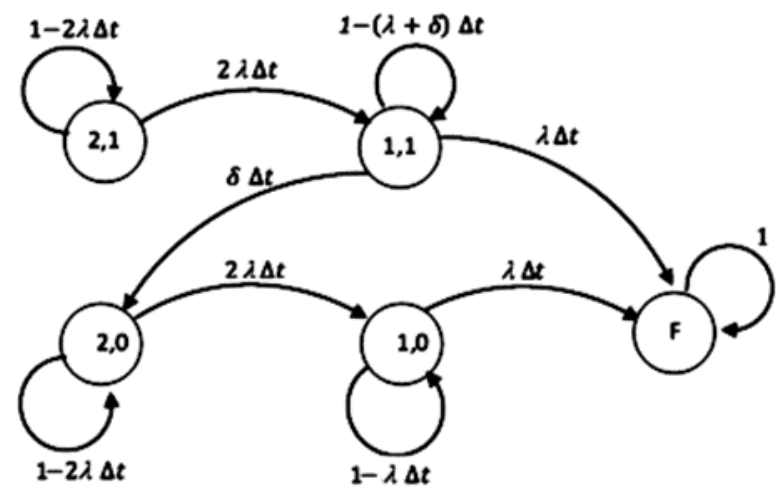

Figure 5: Graph of Markov chain in hybrid cloud service C
In Hybrid cloud service model in four states is normal and gives service thus the possibility of the occurrence of each step can be calculated and in order to evaluate the reliability of hybrid cloud service $\mathrm{C}$ from the Markov model (Figure 5) the steady state transition matrix of this hybrid cloud service can be written as Equation (1).

$P=\left[\begin{array}{ccccc}-2 \lambda & 2 \lambda & 0 & 0 & 0 \\ 0 & -(\lambda+\delta) & \delta & 0 & \lambda \\ 0 & 0 & -2 \lambda & 2 \lambda & 0 \\ 0 & 0 & 0 & -\lambda & \lambda \\ 0 & 0 & 0 & 0 & 0\end{array}\right]$

Assuming the initial conditions as Equation (1) and solving the matrix transpose, the probability of normal activities of the root service cloud can be expressed as Equations (2).

$$
\begin{aligned}
& \mathrm{P}_{1}(0)=0, \mathrm{P}_{2}(0)=0, \mathrm{P}_{3}(0)=0, \mathrm{P}_{4}(0)=0, \mathrm{P}_{\mathrm{F}}(0)=0 \\
& \mathrm{P}_{1}=\mathrm{e}^{-2 \lambda \mathrm{t}} \\
& \mathrm{P}_{2}=\frac{2 \lambda}{\lambda-\delta}\left(\mathrm{e}^{-(\lambda+\delta) t}-\mathrm{e}^{-2 \lambda \mathrm{t}}\right) \\
& \mathrm{P}_{3}=\frac{2 \lambda \delta}{(\lambda-\delta)^{2}}\left(\mathrm{e}^{-(\lambda+\delta) \mathrm{t}}-\mathrm{e}^{-2 \lambda \mathrm{t}}+(\lambda-\delta) \mathrm{te}^{-2 \lambda t}\right) \\
& \mathrm{P}_{4}=\frac{4}{\lambda-\delta}\left(\lambda \delta \mathrm{t} \mathrm{e}^{-2 \lambda \mathrm{t}}+\left(\delta+\frac{\lambda \delta}{(\lambda-\delta)}\right) \mathrm{e}^{-2 \lambda t}-\lambda^{2} \mathrm{e}^{-(\lambda+\delta) \mathrm{t}}+\right. \\
& \left.\left(\lambda^{2}-\frac{\lambda \delta}{(\lambda-\delta)}-\delta\right) \mathrm{e}^{-\lambda \mathrm{t}}\right)
\end{aligned}
$$

Reliability can be calculated from the sum of 2 to 5 Equations and Equation (6) is obtained from summing the above equations.

$$
\begin{aligned}
\mathrm{R}_{\text {Cloud }-\mathrm{C}=\mathrm{P}_{1}+\mathrm{P}_{2}} & +\mathrm{P}_{3}+\mathrm{P}_{4} \\
=\mathrm{e}^{-2 \lambda \mathrm{t}}+ & \frac{2 \lambda}{\lambda-\delta}\left(\mathrm{e}^{-(\lambda+\delta) \mathrm{t}}-\mathrm{e}^{-2 \lambda \mathrm{t}}\right) \\
& +\frac{2 \lambda \delta}{(\lambda-\delta)^{2}}\left(\mathrm{e}^{-(\lambda+\delta) \mathrm{t}}-\mathrm{e}^{-2 \lambda \mathrm{t}}\right. \\
& \left.+(\lambda-\delta) \mathrm{te}^{-2 \lambda \mathrm{t}}\right) \\
& +\frac{4}{\lambda-\delta}\left(\lambda \delta \mathrm{t}^{-2 \lambda t}+\left(\delta+\frac{\lambda \delta}{(\lambda-\delta)}\right) \mathrm{e}^{-2 \lambda t}\right. \\
& -\lambda^{2} \mathrm{e}^{-(\lambda+\delta) \mathrm{t}} \\
& \left.+\left(\lambda^{2}-\frac{\lambda \delta}{(\lambda-\delta)}-\delta\right) \mathrm{e}^{-\lambda \mathrm{t}}\right)
\end{aligned}
$$

Reliability of roots hybrid cloud service in Equation (7) after simplification can be expressed as Equation (8).

$\mathrm{R}_{\text {Cloud }-\mathrm{C}}=\frac{6 \lambda \delta}{(\lambda-\delta)} \mathrm{te}^{-2 \lambda \mathrm{t}}+\left(\frac{2 \lambda \delta}{(\lambda-\delta)^{2}}+\frac{3 \delta-\lambda}{(\lambda-\delta)}\right) \mathrm{e}^{-2 \lambda \mathrm{t}}+$
$\left(\frac{\delta}{(\lambda-\delta)^{2}}+\frac{1-2 \lambda}{(\lambda-\delta)}\right) 2 \lambda \mathrm{e}^{-(\lambda+\delta) \mathrm{t}}+\frac{4}{\lambda-\delta}\left(\lambda^{2}-\frac{\lambda \delta}{(\lambda-\delta)}-\delta\right) \mathrm{e}^{-\lambda \mathrm{t}}$

Also, Equation (9) is used to express MTTF.

MTTF $_{\text {Cloud }-\mathrm{C}}=$
$\frac{-3 \delta(2 \lambda \mathrm{t}+1)}{2 \lambda(\lambda-\delta)} \mathrm{e}^{-2 \lambda \mathrm{t}}-\left(\frac{\delta}{(\lambda-\delta)^{2}}+\frac{3 \delta-\lambda}{2 \lambda(\lambda-\delta)}\right) \mathrm{e}^{-2 \lambda \mathrm{t}}-\left(\frac{\delta}{(\lambda+\delta)(\lambda-\delta)^{2}}+\right.$
$\left.\frac{1-2 \lambda}{\left(\lambda^{2}-\delta^{2}\right)}\right) 2 \lambda \mathrm{e}^{-(\lambda+\delta) \mathrm{t}}-\frac{4}{\lambda-\delta}\left(\lambda-\frac{\delta}{(\lambda-\delta)}-\frac{\delta}{\lambda}\right) \mathrm{e}^{-\lambda \mathrm{t}}$

If in Equation (8) we consider the value of $\delta$ zero, then the reliability of root cloud service is expressed as Equation (9). In fact, the possibility of replacing and activity of cloud service reservation as a sub-service is zero and hybrid cloud service $\mathrm{C}$ along with two sub- cloud services of $\mathrm{A}, \mathrm{B}$ activates as redundant and parallel.

$\mathrm{R}_{\text {Cloud }-\mathrm{C}}=2 \mathrm{e}^{-\lambda \mathrm{t}}-\mathrm{e}^{-2 \lambda \mathrm{t}}$ 
When service disruptions occur in cloud federation, it is necessary to recognize the defective part of cloud as soon as possible, so that other providers who work with the same service are not penalized unnecessarily.

\section{SIMULATION}

In this section, simulation of add-on combined cloud service has been done. Concerning figure 5, failure and QoS arrays have been created for combined cloud service $\mathrm{C}$ and two cloud sub-services A and B.

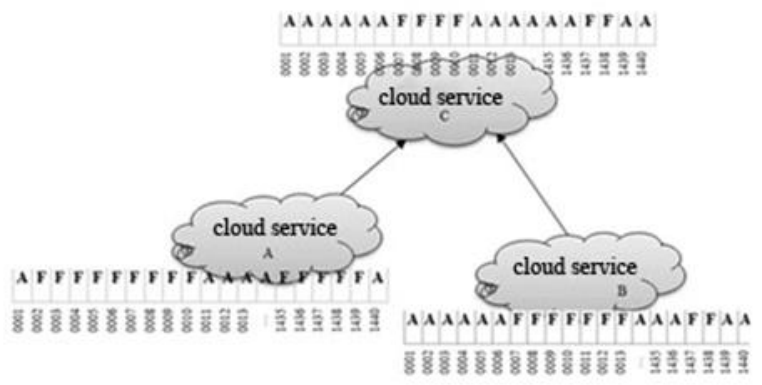

Figure5. Simulation of add-ons combined cloud service

By application of A and B cloud services as add-ons, the mean failure rate has increasingly decreased and as can be seen in figure 6 , the comparison of reliability figure of cloud services $\mathrm{A}$ and $\mathrm{B}$ and combined cloud service $\mathrm{C}$ shows that the reliability of $\mathrm{C}$ cloud has increasingly improved and properly increased.

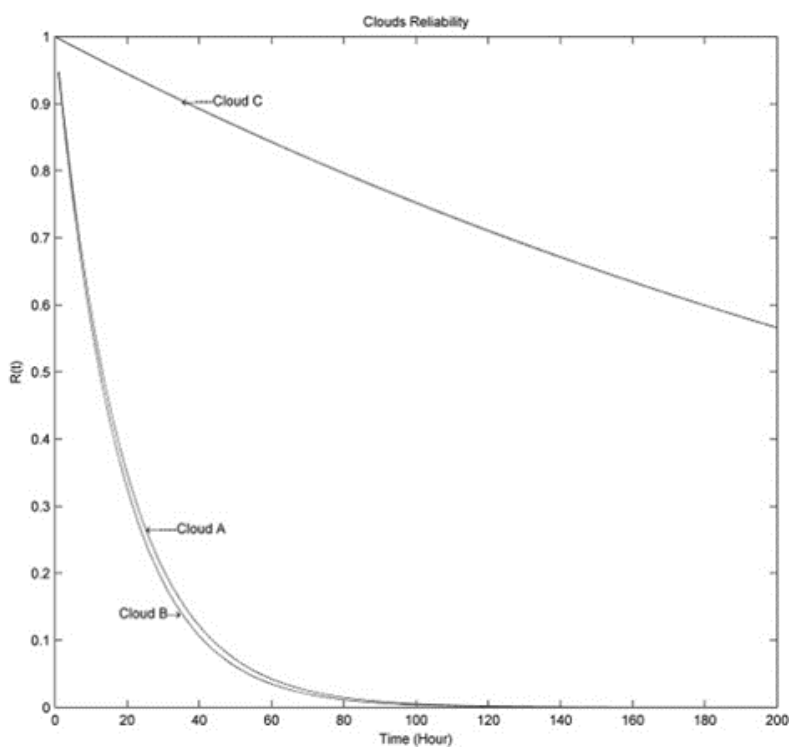

Figure6: The comparison of the reliability of A, B and C cloud services

Following, we performed simulation of add-ons combined cloud and bottleneck cloud. In this scenario, it is assumed that $\mathrm{CM}$ has mean failure rate of $10 \%$, monthly, and for reduction of failure rate and improvement of QoS array, it is possible to transform it to similar combined cloud service SP.

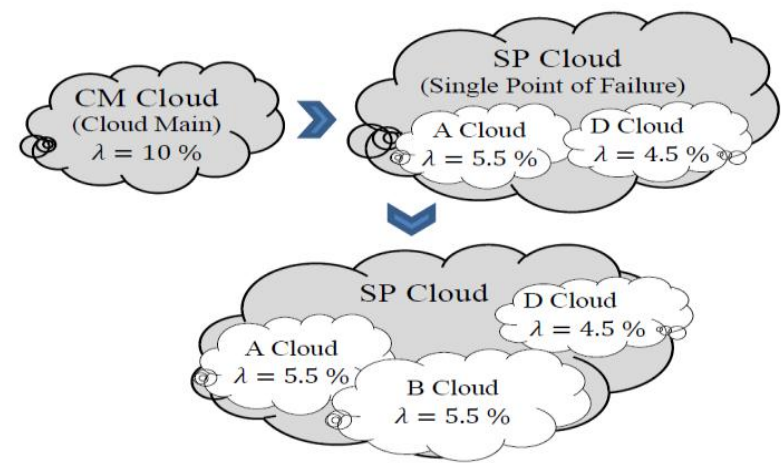

Figure7: The use of SP cloud instead of CM

Failure and QoS arrays were created for CM services and three sub-services of A, B and D was created. Then, the arrays of failure item for each cloud have been created with mean values of failure and registered.

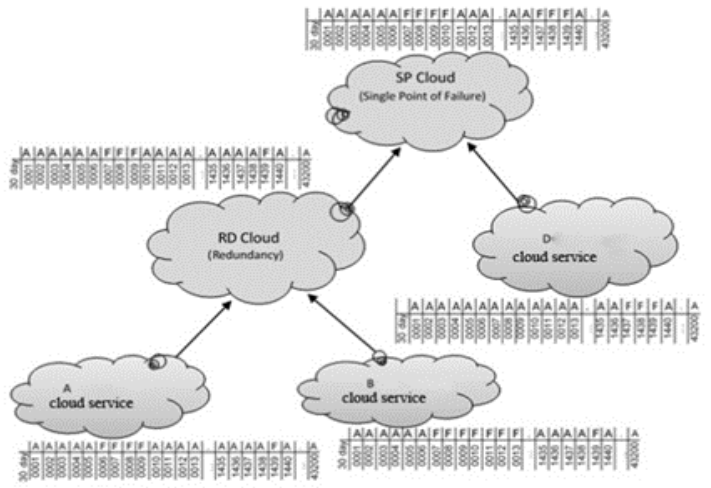

Figure8: The tree of add-ons combined and SP bottleneck cloud

The analysis of CM failure array of CM cloud, the mean value of failure rate, MTTF, MTBF and R(t) have been computed daily and registered in QoS array by simulator. Figure 9 represents the daily mean failure figure, MTTF and MTBF for one month. Moreover, mean failure of one month was computed as $9.92824 \%$, mean MTTF as 9.33591 minutes and mean MTBF as 10.33591 minutes that are shown in figure 9 . The reliability has been computed with mean failure rate by simulator and its $R(t)$ graph shown in figure 9 using $R(t)=e^{-\lambda t}$ equation.

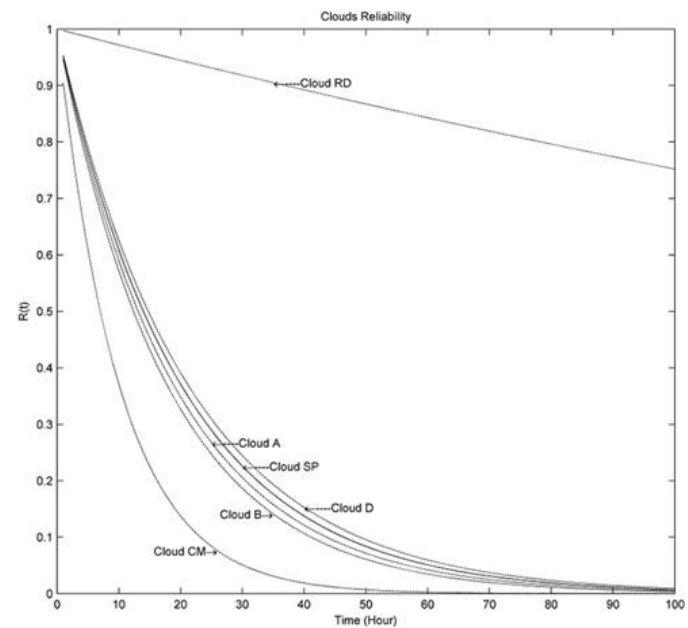

Figure9: The comparison of the reliability of A, B and C cloud services 


\section{CONCLUSION AND FUTURE WORK}

The present paper through using a dynamic measurements mechanism provided an appropriate framework for the calculation of service quality parameters e.g. average failure rate and reliability, for each single cloud service and then root hybrid cloud service. The simulation results of this measurement mechanism compared to the type of cloud service connectivity in hybrid cloud service showed improvement in the parameters of the quality of hybrid cloud service.The present framework was an appropriate one to calculate QoS parameters consisting the methods of calculating and measuring the average failure rate, MTTF, MTBF and reliability in cloud federation for all sub-clouds of hybrid cloud service. The method which in this this framework was used to measure the service quality of hybrid cloud, was based on the behavior recording and overall performance of cloud service that through analyzing the recording of each sub- cloud service related to a hybrid cloud service its service quality was calculated. One of the important advantages of our method is the possibility to measure service quality continuously. Another advantage of this mechanism is the structure of a tree service, since on one hand provides the possibility of extensibility and on the other hand provides recursive calculations which reduce the complexity of software and code writing.

\section{REFERENCES}

[1] Celesti, Antonio, et al. "Virtual machine provisioning through satellite communications in federated cloud environments." Future Generation Computer Systems 28.1 (2012): 85-93.

[2] Ahson, Syed A., and Mohammad Ilyas, eds. Cloud computing and software services: Theory and techniques. CRC Press, 2010.

[3] Wang, Guijun, et al. "Compositional QoS Modeling and Analysis of Cloud-based Federated Ecosystems." Enterprise Distributed Object Computing Conference (EDOC), 2012 IEEE 16th International. IEEE, 2012.
[4] Hershey, Paul C., et al. "System of Systems for Qualityof-Service Observation and Response in Cloud Computing Environments." 1-11.

[5] Khazaei, Hamzeh. Performance modeling of cloud computing centers. Diss. The University of Manitoba, 2013.

[6] Dew, Peter M., and Shahzad Nizamani. "QAComPS: a quality-aware federated computational semantic web service for computational modellers." URL http://weblidi. info. unlp. edu. ar/worldcomp2011mirror/SWW3720. pdf.

[7] Frey, Stefan, et al. "Fuzzy Controled QoS for Scalable Cloud Computing Services." CLOUD COMPUTING 2013, The Fourth International Conference on Cloud Computing, GRIDs, and Virtualization. 2013.

[8] Ferretti, Stefano, Vittorio Ghini, Fabio Panzieri, Michele Pellegrini, and Elisa Turrini. "Qos-aware clouds." In Cloud Computing (CLOUD), 2010 IEEE 3rd International Conference on, pp. 321-328. IEEE, 2010.

[9] Celesti, Antonio, et al. "Virtual machine provisioning through satellite communications in federated cloud environments." Future Generation Computer Systems 28.1 (2012): 85-93

[10] Youseff, Lamia, et al. "Understanding the cloud computing landscape." Cloud Computing and Software Services (2010): 1.

[11] Velte, Toby, Anthony Velte, and Robert Elsenpeter. Cloud computing, a practical approach. McGraw-Hill, Inc., 2009.

[12] Armbrust, Michael, et al. "A view of cloud computing." Communications of the ACM 53.4 (2010): 50-58.

[13] T OGRAPH, B., and Y. RICHARD MORGENS. "Cloud computing."Communications of the ACM 51.7 (2008). 